\title{
A precise location navigation map visualization method for landmark guidance
}

\author{
Wang haiying ${ }^{1,3}$, Yan haowen ${ }^{1,2, *}$ \\ 1 Faculty of Geomatics, Lanzhou Jiaotong University, Lanzhou 730070, China - (121619581@qq.com, haowen2010@gmail.com) \\ 2 Gansu Provincial Engineering Laboratory for National Geographic State Monitoring, Lanzhou 730070, China \\ 3 The Institution of Surveying and mapping in Lanzhou City, Lanzhou 730070, China
}

Keywords: precise location, navigation map, landmark guidance

\begin{abstract}
:
Due to the errors of the global positioning system and the navigation map, the navigation map can only locate the approximate location of the points of interest on the map, which causes the navigation task to end at a distance of several meters to several tens of meters from the real destination. The navigation of the left distance needs to be done by the user himself. In addition, the number of points of interest on the navigation map is limited, and the user can only navigate to the points of interest near the destination and then complete the remaining navigation by himself. This paper proposes a navigation map guided by landmarks, where the user finds the exact location of the destination. The map has the following characteristics: 1) The map is produced by general users 2) The map can be displayed globally or in segments 3) Highlight landmarks 4) Map navigation does not depend on GPS 5) Simple spread
\end{abstract}

1. There is a navigational deviation of the points of interest on the map, and the navigation diagram is used to highlight the relationship between the parking lot gate and the road

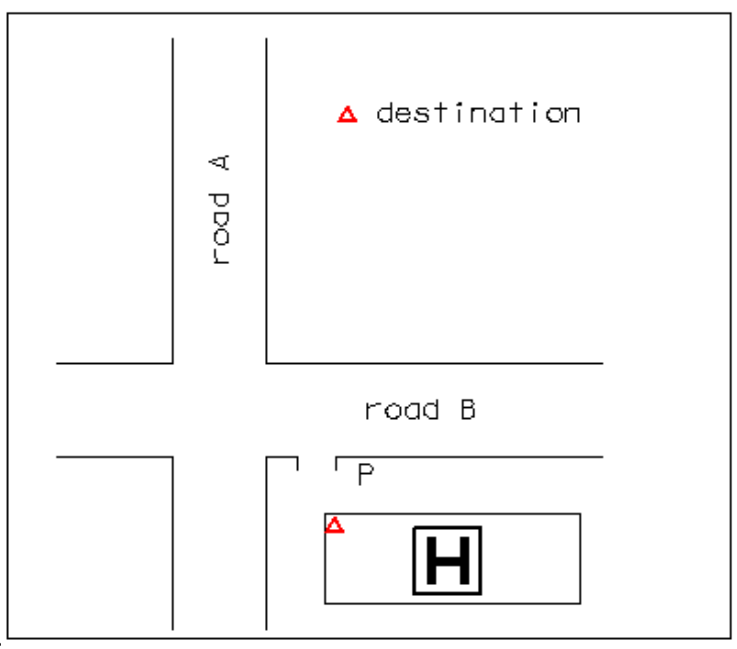

Figure 1. The entrance location of a hotel parking lot, roadB is a one-way street

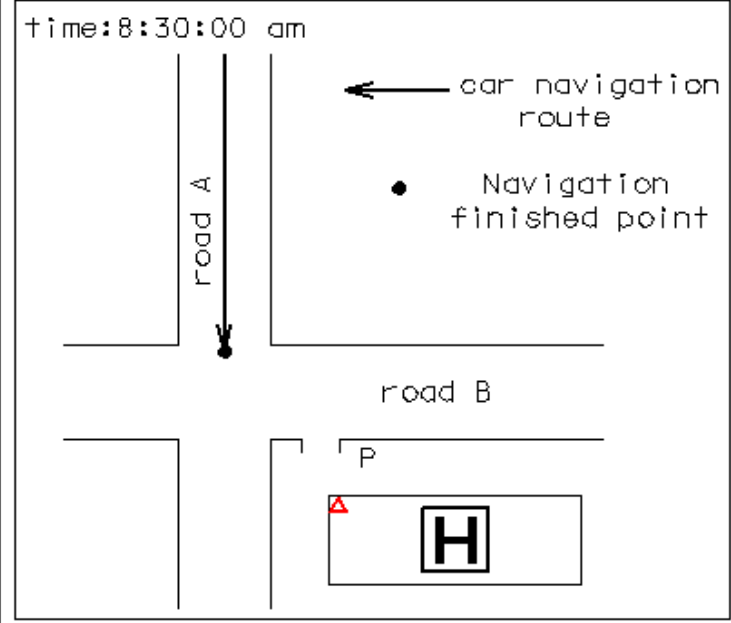

Figure 2. Show the location of the vehicle at the end of the navigation 


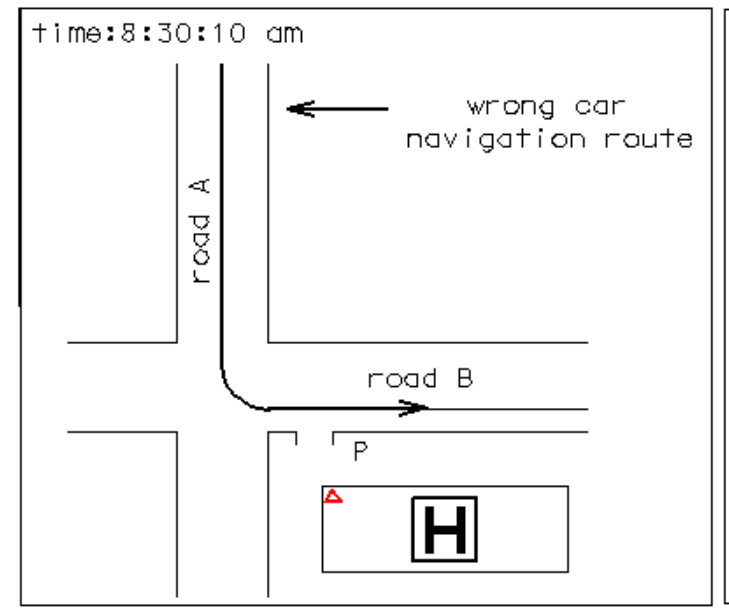

Figure 3. The car has continued to travel after the navigation ends, has missed the hotel parking entrance

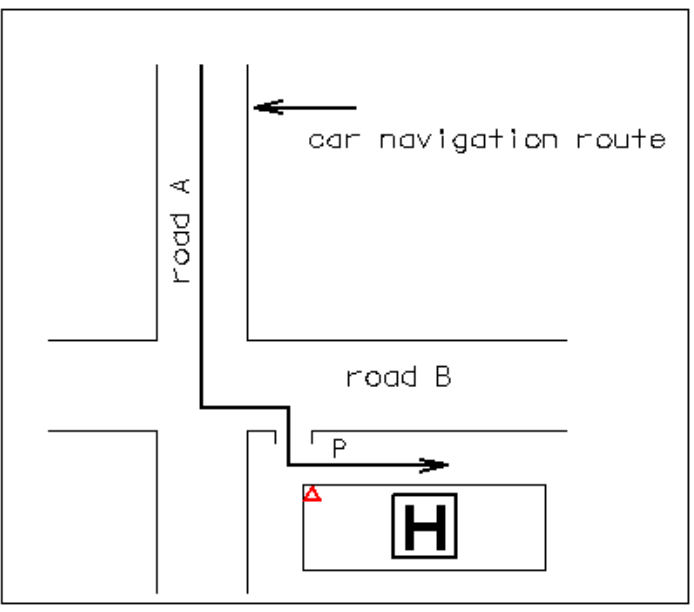

Figure 4. Hotel parking lot entrance navigation map

2. Create a navigation map for points of interest that are not on the navigation map

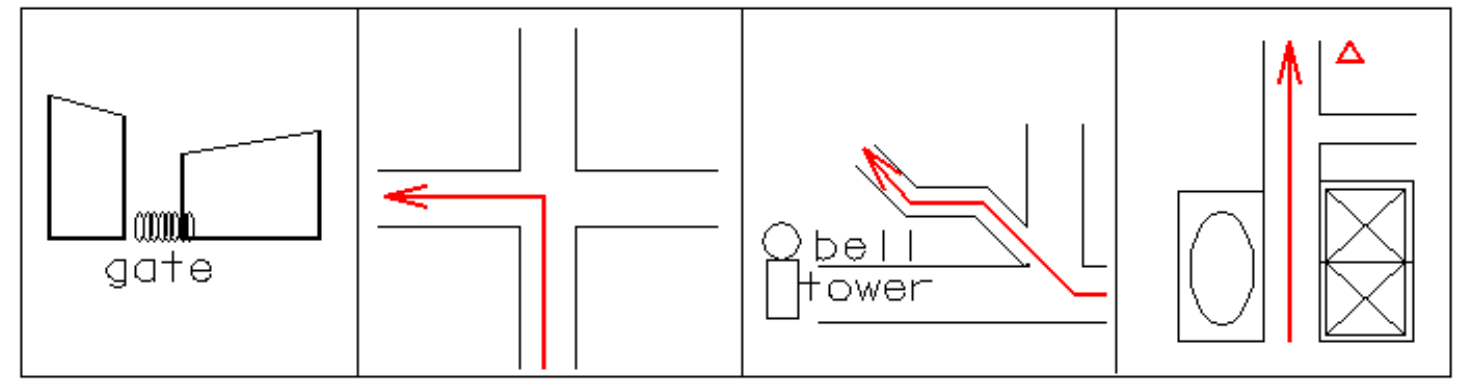

Figure 5. A map of a home address drawn by someone, segmented display 


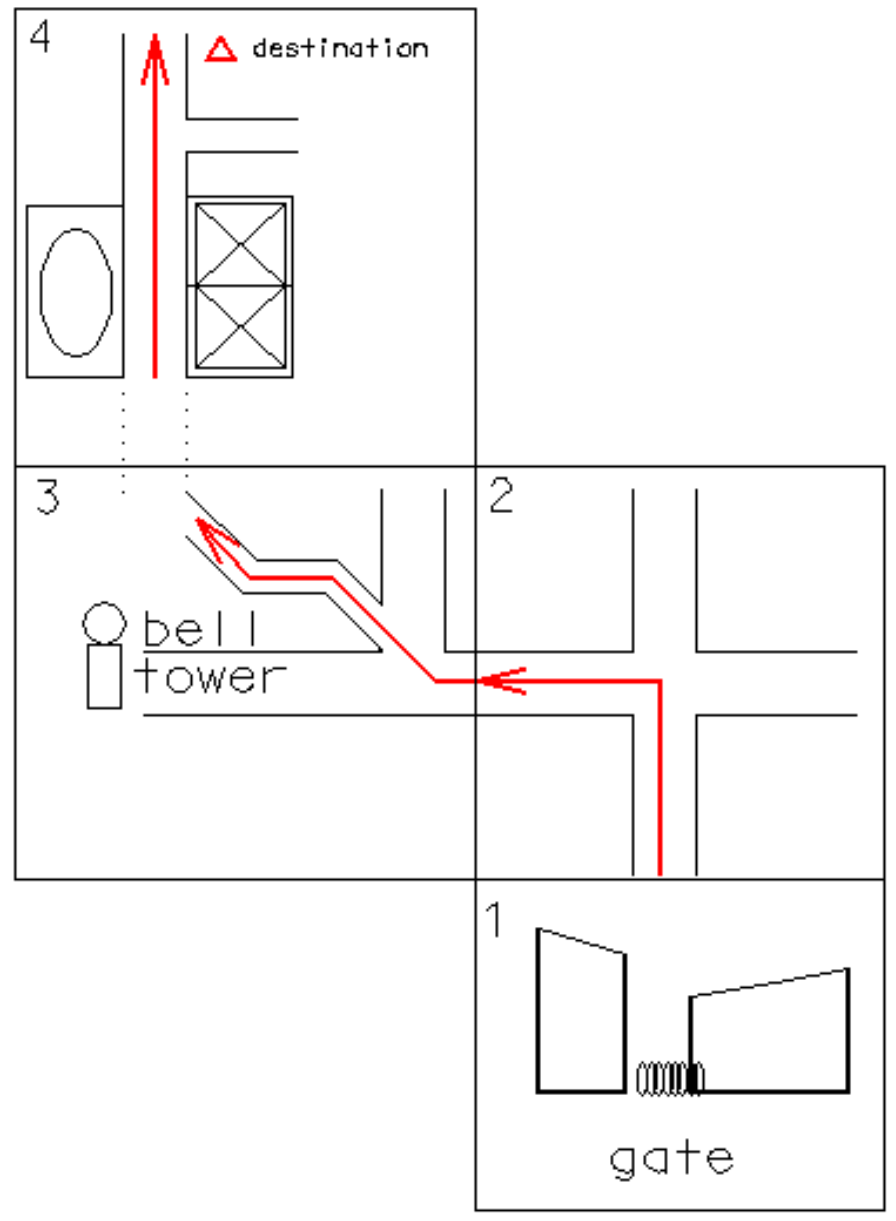

Figure6. A map of a home address drawn by someone, global display 